\title{
Co-ferrite Thin Films with Perpendicular Magnetic Anisotropy
}

\author{
Xiaoxi Liu, Sagar Shirasath, and Kensuke Shindoh \\ Faculty of Engineering, Shinshu University, Wakasato 4-17-1, Nagano 380-8553, Japan
}

\begin{abstract}
Co-ferrite thin film with spinel structure is attractive for spintronics and multifunctional devices. So far, single crystal substrates are necessary to introduce perpendicular magnetic anisotropy in Co-ferrite films. In this paper, a unique process has been developed to deposit Co-ferrite thin films onto thermally oxidized silicon wafer with perpendicular magnetic anisotropy. We have successfully prepared Co-ferrite films with $(00 l)$ orientation. Those films show perpendicular coercivities as large as $12.8 \mathrm{kOe}$. X-ray diffractometry results revealed that compressive strain, which is introduced by the post-annealing process rather than deposition process, is as large as $1.3 \%$ in films with $(00 l)$ orientation. The large coercivity and perpendicular magnetic anisotropy can be quantitatively explained by the magneto-elastic effect. This silicon compatible fabrication process is useful to further extending the applications of Co-ferrite films.
\end{abstract}

Index Terms - perpendicular magnetic anisotropy, Co-ferrite, silicon compatible fabrication, magneto-elastic effect.

\section{INTRODUCTION}

$\mathrm{C}$ O-FERRITE with spinel structure is one of the attractive materials for spintronics and artificial multiferroics. Due to the insulating and high-curie temperature properties, Coferrite thins films have potential applications in such so called spin filters, where a combination of insulating ferrite with a non-magnetic electrode is believed capable of creating pure spin polarized currents [1]. Co-ferrite also shows large magnetostrictive effect. Which can be used for enhancing magnetoelectric coupling between ferroelectric phase and magnetic phase of multiferroic nanostructures [2]. Films with $(00 l)$ orientation and perpendicular magnetic anisotropy are essential to realize the interesting properties of Co-ferrite thin films. So far, Single crystal substrate such as $\mathrm{SrTiO}_{3}$ [2], $\mathrm{MgO}$ [3] have been reported for the heteroepitaxial growth of $(00 l)$ orientated Co ferrite films. However, from the practical application point of view, it is strongly recommended to prepare orientated Co ferrite films onto thermally oxidized silicon wafers $\left(\mathrm{SiO}_{2} / \mathrm{Si}\right)$, which have amorphic surfaces .

In this experiment, a unique process has been developed to deposit Co-ferrite thin films onto thermally oxidized silicon wafer with perpendicular magnetic anisotropy by facing target sputtering [4]. Co-ferrite films with preferential (00l) orientation have been successfully prepared. Those films show coercivity in the perpendicular direction as large as $12.8 \mathrm{kOe}$. Of particular interest is that the perpendicular magnetic anisotropy and coercivity of the films are depended on the oxygen content in the films. Three dimensional lattice parameters determined by in-plane X-ray diffractometry to be $\mathrm{a}=\mathrm{b}=8.43 \AA, \mathrm{c}=8.35 \AA$. Which indicate a compressive out-ofplane strain as large as $1.1 \%$.

\section{EXPERIMENTAL PROCEDURE}

In this study, Co-ferrite thin films were prepared by a facing targets sputtering (FTS) system. Co-ferrite disks with stoichiometric composition are used as targets. The films are deposited by a two-steps process. A thin layer (less than the $30 \%$ of the total film thickness) was first deposited onto

Manuscript received March 20, 2015. Corresponding author: X. Liu (email: liu@cs.shinshu-u.ac.jp).

Digital Object Identifier inserted by IEEE thermally oxidized substrate without substrate heating. A seconded layer was deposited after the substrate temperature was elevated up to $250{ }^{\circ} \mathrm{C}$. Pure Ar gas was used as sputtering gas. Unlike the normal process to prepare ferrite films [4], oxygen gas was not introduced into the sputtering chamber during the sputtering process. The prepared films were crystallized and show moderate coercivity. The films were further annealed in oxygen to improve the coercivity. We found about $20 \%$ increase of film thickness due to the annealing process. In this experiment, we used X-ray diffractometry (XRD) and transmission electron microscopy (TEM) to determine crystal structures of the films. $\theta-2 \theta$ scan $\mathrm{XRD}$ was used to detect the film orientation and the lattice constants normal to the film plane, while in-plane XRD was used to obtain in-plane lattice constants. The magnetic properties, magnetic domains, surface morphology, chemical status of the films were characterized by vibrating sample magnetometry (VSM), magnetic force microscopy (MFM), scanning electron microscopy (SEM), and X-ray photoelectron spectroscopy (XPS).

\section{RESULTS AND DISCUSSION}

Fig. 1 shows $\theta-2 \theta$ scan XRD diagrams of films deposited without substrate heating (a), and films deposited at substrate temperature of $250{ }^{\circ} \mathrm{C}$ (b), respectively. Films, with thickness

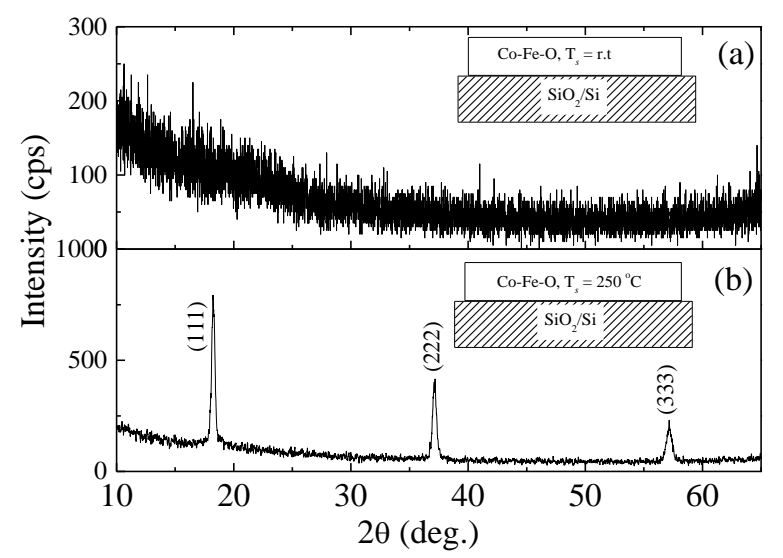

Fig. 1. $\theta-2 \theta$ scan XRD diagrams of films deposited without substrate heating (a), and films deposited at substrate temperature of $250{ }^{\circ} \mathrm{C}(\mathrm{b})$. 


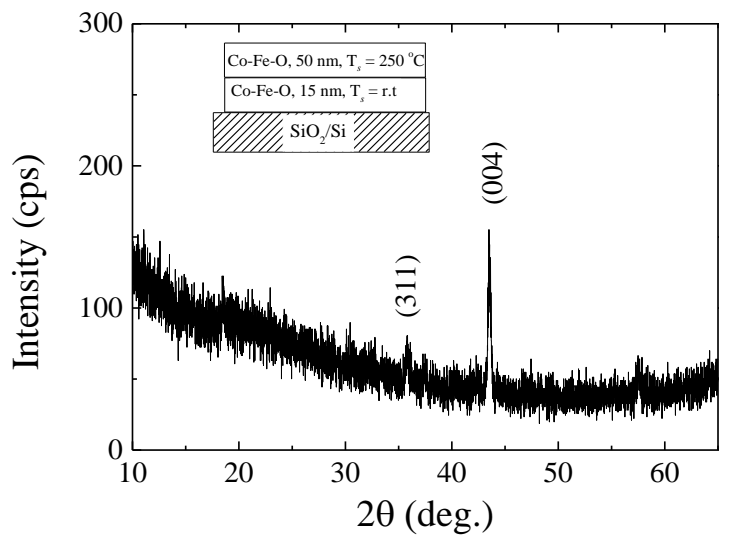

Fig. 2. $\theta-2 \theta$ scan XRD diagrams of films deposited by a two-step process.

of $50 \mathrm{~nm}$, were deposited at sputtering gas pressure of $0.5 \mathrm{~Pa}$. As show in (a), films deposited without substrate heating show $\mathrm{X}$-ray amorphic structure with no obvious diffraction lines in the XRD diagram. However, films deposited at $250{ }^{\circ} \mathrm{C}$ show strong diffraction lines from (111), (222) and (333) plane.
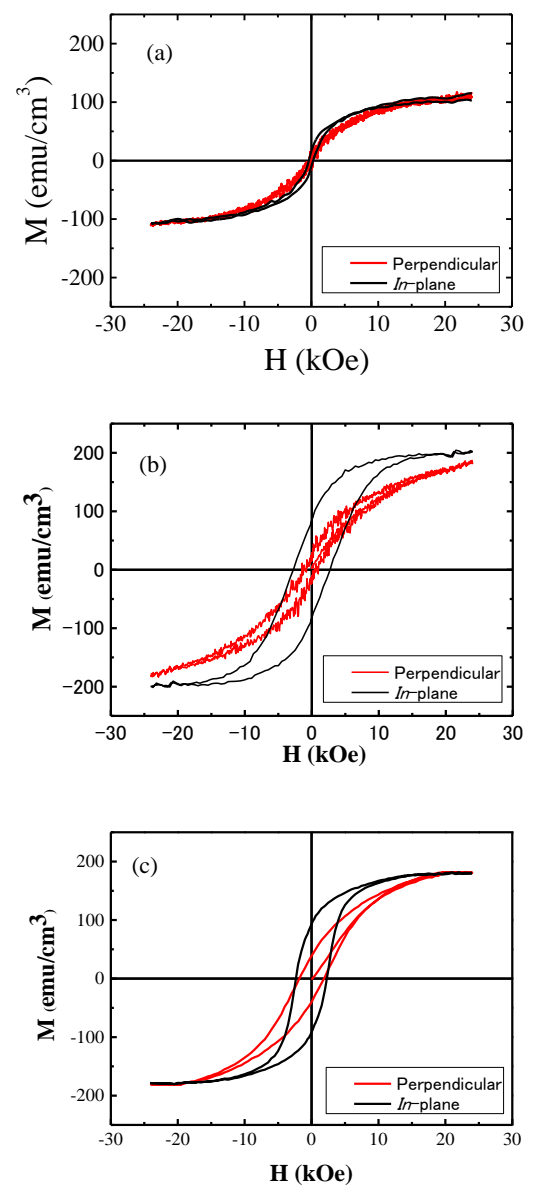

Fig. 3. Hysteresis loops of as-deposited films. (a) films deposited without substrate heating; (b) films deposited at substrate temperature of $250{ }^{\circ} \mathrm{C}$; (c) films deposited by the two-step process.
Since (111) plane is the most close packed plane in cubic spinel structure, fig. 1(b) means the films deposited at $250{ }^{\circ} \mathrm{C}$ show (111) orientation.

Fig. 2 shows $\theta-2 \theta$ scan XRD diagrams of films deposited by a two-step process, a $15 \mathrm{~nm}$ thick films was first deposited without substrate heating, a $50 \mathrm{~nm}$ thick films was deposited subsequently after the substrate temperature elevated up to $250{ }^{\circ} \mathrm{C}$. According to fig. 2, films deposited by the above twostep process show quite different XRD diagrams as compared to films deposited by the one-step process as shown in fig. 1. The films show (004) diffraction line as the strongest diffraction line. We can see only a weak (311) diffraction line, which is the strongest line in powder diffraction file of Coferrite. These results indicate the films have preferential $(00 l)$ orientation.

Fig. 3 shows hysteresis loops of as deposited films. (a) films deposited without substrate heating; (b) films deposited at substrate temperature of $250{ }^{\circ} \mathrm{C}$; (c) films deposited by the two-step process. According to fig. 3(a), films deposited without substrate heating show weak soft magnetic properties with low saturation magnetization and small coercivity in both perpendicular and in-plane directions. An increase of saturation magnetization was found for films deposited at an elevated substrate temperature as shown in fig. 3 (b) and (c). Both films show moderate coercivities. However, the magnetic anisotropy in films (b) and (c) are slightly different. Films deposited by one-step process show clearly in-plane magnetic anisotropy with larger perpendicular coercivities,

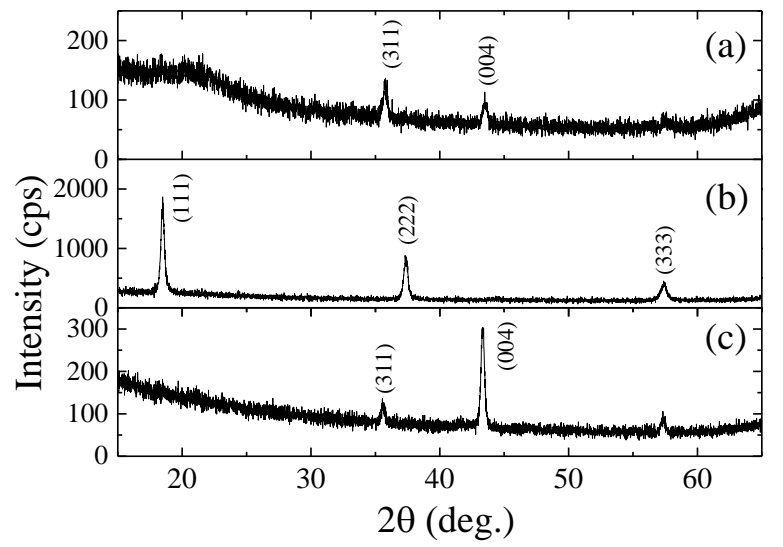

Fig. 4. $\theta-2 \theta$ scan XRD diagrams of various post annealed films with annealing temperature at $800{ }^{\circ} \mathrm{C}$ for one hour. The films are (a) films deposited without substrate heating; (b) films deposited at substrate temperature of $250{ }^{\circ} \mathrm{C}$; (c) films deposited by the two-step process.

and smaller in-plane coercivities, while films deposited by two-step process show slightly higher in-plane coercivites.

Both crystal structure and magnetic properties of the films are characterized after annealing at ambient circumstance at different temperatures. We have fund improvement of crystal structure and magnetic properties after annealing the samples above $600{ }^{\circ} \mathrm{C}$. Fig. 4 shows XRD diagrams of various post annealed films with annealing temperature at $800{ }^{\circ} \mathrm{C}$ for one hour. The films are (a) films deposited without substrate heating; (b) films deposited at substrate temperature of $250{ }^{\circ} \mathrm{C}$; (c) films deposited by the two-step process. Dramatically 
increases of diffraction intensities were found for all the samples. This suggests that the crystallinity of all the samples were improved after annealing. Films deposited without substrate heating (a) show random orientation with (311) as the strongest diffraction line. Films deposited at $250{ }^{\circ} \mathrm{C}$ show clearly (111) orientation. While films prepared by the two step process show (004) preferential orientation. It should be noted that the crystal orientation are almost the same as the as-
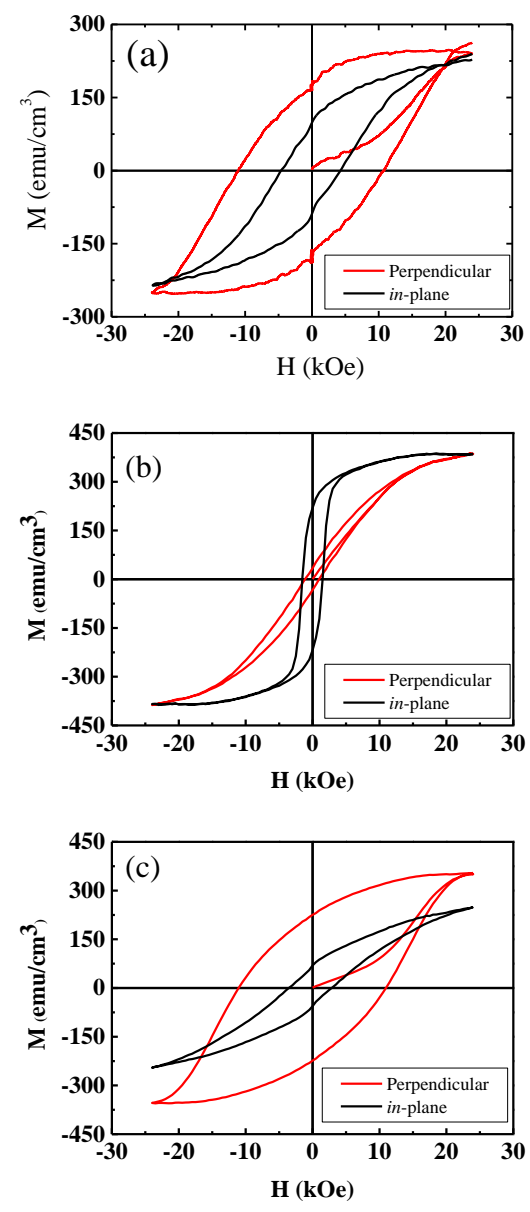

Fig. 5. Hysteresis loops of various post annealed films with annealing temperature at $800{ }^{\circ} \mathrm{C}$ for one hour. (a) films deposited without substrate heating; (b) films deposited at substrate temperature of $250{ }^{\circ} \mathrm{C}$; (c) films deposited by the two-step process.

deposited films as show in fig. 1 and fig. 2 except the films deposited without substrate heating.

Fig. 5 shows hysteresis loops of various post annealed films with annealing temperature at $800{ }^{\circ} \mathrm{C}$ for one hour. (a) films deposited without substrate heating; (b) films deposited at substrate temperature of $250{ }^{\circ} \mathrm{C}$; (c) films deposited by the two-step process. A huge improve of perpendicular coercivity were found for films deposited without substrate heating (a) and two-step process (c). On the other hand, the coercivities in both perpendicular and in-plane direction slightly decrease with the post annealing process for films deposited at $250{ }^{\circ} \mathrm{C}$ (b).

To understand the mechanism of coercivity improvement by post-annealing in those films have $(00 l)$ normal to the films plane orientation, we have performed both $\theta-2 \theta$ scan XRD

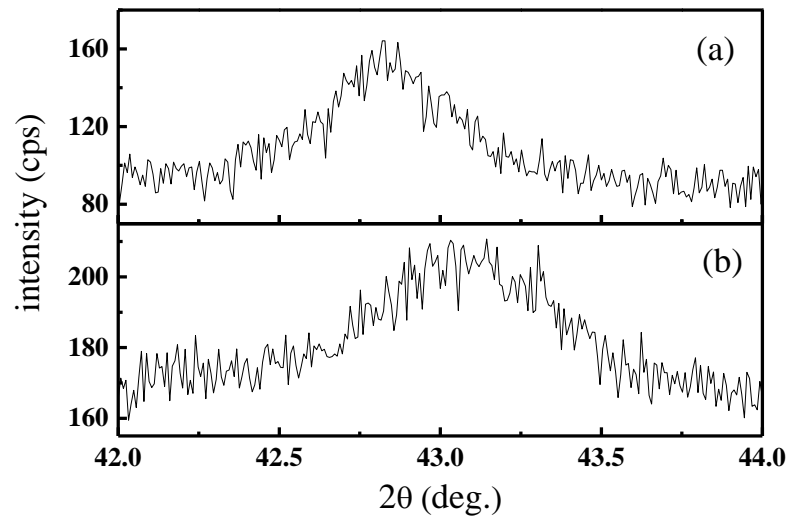

Fig. 6. Diffraction profile of (004) of as-deposited films by two-step process. (a) $\theta-2 \theta$ scan (focusing method); and (b) in-plane .

(Bragg-Brentano focusing method [6]) and in-plane XRD measurement for the films. Fig. 6 shows the diffraction profile of (004) of as-deposited films by two-step process. (a) shows the results of $\theta-2 \theta$ scan (focusing method), and (b) shows the results of in-plane XRD. Normally, $\theta-2 \theta$ scan by focusing method reveals inter-planar spacing of those crystal planes parallel to the films surface, while in-plane XRD results reveals the inter-planar spacing of planes normal to the films surface. Clearly line shift were found for the two different measurements. We have calculated the $3 \mathrm{D}$ spinel lattice parameters of Co-ferrite based on the peak position, which has been fitted by assuming a Gaussian distribution of the diffraction profile. The result is illustrated in Fig. 7. As-

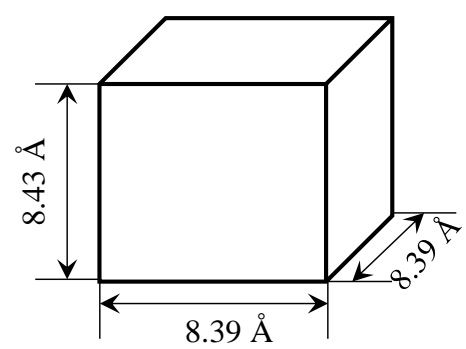

Fig. 7. Illustration of the unit cell of as-deposited Co-ferrite films prepared by two-step process.

deposited films have lattice parameters of $a=b=8.39 \AA$, while $c=8.43 \AA$. Which indicating as-deposited Co-ferrite films have a tensile out-of-plane strain of $0.5 \%$.

Fig. 8 shows the diffraction profile of (004) of films annealed at $800{ }^{\circ} \mathrm{C}$. (a) shows the result of $\theta-2 \theta$ scan (focusing method), and (b) shows the result of in-plane XRD. The calculated 3D spinel lattice parameters were illustrated in fig.9. Post-annealed films have lattice parameters of $a=b=8.43 \AA$, while $c=8.35 \AA$. Which indicating post-annealed Co-ferrite films have a compressive out-of-plane strain as large as $1.3 \%$.

The above results clearly show strain evolution due to postannealing in those $(00 l)$ oriented films. This compressive strain in Co-ferrite films induced by post-annealing can be related to the perpendicular magnetic anisotropy through its magnetoelastic effect. The stress in the $(00 l)$ orientated films 


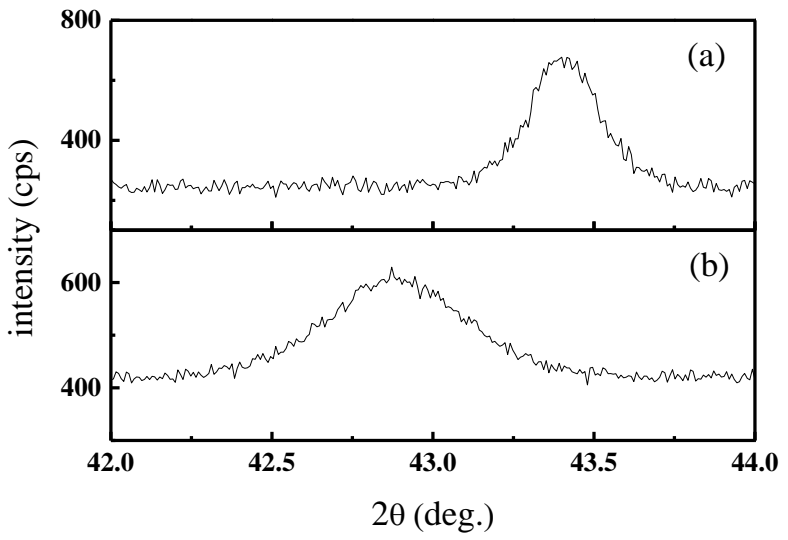

Fig. 8. Diffraction profile of (004) of post-annealed films by two-step process. (a) $\theta-2 \theta$ scan (focusing method); and (b) in-plane .

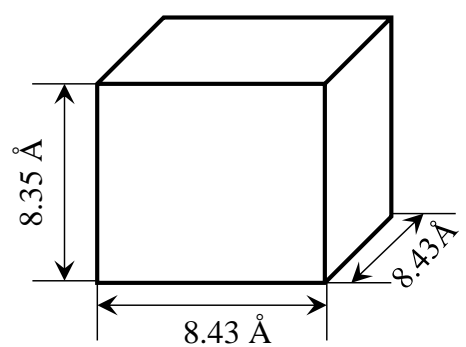

Fig. 9. Illustration of the unit cell of post annealed Co-ferrite films prepared by two-step process.

is given by $\sigma_{00 l}=Y \varepsilon_{00 l}$, in which $\mathrm{Y}$ is Young's modulus ( $141.6 \mathrm{GPa}[5])$ and $\varepsilon_{00 l}$ is the strain along (00l) direction. The magnetoelastic anisotropy energy corresponding to it is $E=-3 \lambda_{00 l} \sigma_{00 l} / 2$, where $\lambda_{00 l}$ is the magnetostriction coefficient of Co-ferrite $\left(\sim 350 \times 10^{-6}\right.$ [5]). The magnetic anisotropy field contributed by the magnetostriction is given by $\mathrm{H}_{\text {stress }}=2 E / \mathrm{M}_{s}=47 \mathrm{kOe}$. Hence it is easy to understand the large coercivity of $12.8 \mathrm{kOe}$ and still not saturate at a maximum applied field of $24 \mathrm{kOe}$ as shown in fig. 5 (c).

\section{CONCLUSION}

In conclusion, we have successfully prepared Co-ferrite films with preferential $(00 l)$ orientation by a two-step process. In this process, we first deposit films without substrate heating followed by deposit the films at an elevated substrate temperature onto thermally oxide silicon wafers. The as deposited films show moderate coercivities. Perpendicular coercivities as large as $12.8 \mathrm{kOe}$ were obtained by postannealing the films. Focusing method and in-plane XRD results revealed that comprehensive strain as large as $1.3 \%$ was introduced by the post-annealing process. The large coercivity and perpendicular magnetic anisotropy in the postannealed films with $(00 l)$ orientation can be quantitatively explained by the magneto-elastic effect.

\section{REFERENCES}

[1] Jean-Baptiste Moussy, "From epitaxial growth of ferrite films to spinpolarized tunneling,” J. Phys.D: Appl. Phys., 46, 143001(2013).
[2] H. Zheng et.al, "Multiferroic $\mathrm{BaTiO}_{3}-\mathrm{CoFe}_{2} \mathrm{O}_{4}$ nanostructures", Science, 303, 661-663, (2004)

[3] T. Niizeki, et.al "Extraordinarily large perpendicular magnetic anisotropy in epitaxially strained cobalt-ferrite $\operatorname{CoxFe}_{3-\mathrm{x}} \mathrm{O}_{4}(001)(\mathrm{x}=$ $0.75,1.0)$ thin films," Appl. Phys. Lett., 103, 162407 (2013).

[4] A. Morisako et.al, "The effect of underlayer for Ba-ferrite sputtered films on c-axis orientation," J. Appl. Phys., 81(8), 4374-4376 (1997).

[5] V. J. Folen, in Landolt-Börnstein, Vol. 3, Part 4b, Magnetic and Other Properties of Oxides and Related Compounds, K.-H. Hellwege, A.M. Hellwege, Eds. Springer, Berlin, Heidelberg, New York, 1970.

[6] A. Taylor, "An Introduction to X-Ray Metallography," John Wiley \& Sons, NewYork, (1945). 